\title{
Nuclear activity and stellar mass in galaxies
}

\section{G. La Mura*, ${ }^{*}$ D. Bindoni, ${ }^{b}$ S. Ciroi, ${ }^{b}$ V. Cracco, ${ }^{b}$ P. Rafanelli, ${ }^{b}$ L. Vaona, ${ }^{b}$ R. D'Abrusco ${ }^{c}$}

${ }^{a}$ Institut für Astro- und Teilchenphysik, Universität Innsbruck

Technikerstrasse 25/8, A-6020 Innsbruck, Austria

${ }^{b}$ Dipartimento di Fisica e Astronomia, Università di Padova

Vicolo dell'Osservatorio 3, I-35122 Padova, Italy

${ }^{c}$ High Energy Astrophysics Division, Harvard-Smithsonian Center for Astrophysics

60 Garden Street, Cambridge, Massachusetts

E-mail:

giovanni.lamura@unipd.it, daniele.bindoni@unipd.it,

stefano.ciroidunipd.it, valentina.craccolunipd.it,

piero.rafanelli@unipd.it, luigi.vaonadistruzione.it,

rdabruscodcfa.harvard.edu

It is widely believed that the correlations between Supermassive Black Holes and host galaxies descend from a connection between black hole growth and stellar mass assembly. Here we present the results of our investigation on star formation and nuclear activity, studying the relations between the circum-nuclear environments of both active and quiescent galaxies. We find that the mass of the stellar component is a critical parameter that we have to consider to gain further insight into the connection between AGN and star formation processes.

Nuclei of Seyfert galaxies and QSOs - Central engine \& conditions of star formation November 6-8, 2012

Max-Planck-Insitut für Radioastronomie (MPIfR), Bonn, Germany

*Speaker. 


\section{Introduction}

It is now well established that Super Massive Black Holes (SMBH) reside in the nuclei of massive galaxies, including our own. Accretion of matter into their gravitational field is a very effective energy source, which lies at the basis of the extremely powerful and varied phenomenology of Active Galactic Nuclei (AGN). According to recent statistical investigations, approximately 10 per cent of nearby galaxies show hints of nuclear activity, mainly through the presence of emission lines produced by an ionized gas close to the central source (within a region of size $r<1 \mathrm{kpc}$ ). Similarly, another 10 per cent of galaxies are characterized by emission line spectra that can be accounted for by means of photo-ionization from the radiation of young massive hot stars (Taniguchi 2003). In principle, it is possible to distinguish the signature of the two different possibilities from the spectrum emitted by the ionized gas (see e. g. Veilleux \& Osterbrock 1987; Kewley et al. 2006). The task, however, is not straightforward, partially because of the difficulties related to the spectroscopic measurements at the basis of the technique. Moreover, several objects appear to be characterized by intermediate properties.

Though some kind of connection between nuclear activity and star formation is expected, its physical nature is not yet clarified. Many theories were developed, mainly invoking effects that could lead from nuclear star formation to AGNs and then evaluating the energetic feedback on the surrounding medium. However, the possibility that the relation may change, depending on the host galaxy properties, should also be taken into account. Indeed, it has been pointed out that the high velocity outflows, produced by the most massive stars of a young stellar population, are very unlikely to feed nuclear activity and they can even sweep the interstellar medium of low mass galaxies, preventing the onset of further star formation or nuclear activity. In very massive galaxies, on the contrary, the large amount of gas trapped in the gravitational field could serve as fuel for a powerful AGN, which is again expected to stop the formation of new stars (Monaco \& Fontanot 2005; Feruglio et al. 2010, and references therein). Such effects may explain why star formation is currently observed over a typically limited range of medium mass galaxies.

Here we present the results of our study on the properties of the circum-nuclear regions of galaxies, whose spectra indicate the existence of an interstellar medium (ISM), which is ionized either by hot young stars or by non-thermal activity of the nucleus. Comparing such properties with those of quiescent galaxies, we find additional evidence of close relationships between nuclear activity and star formation processes, together with some sequences, that indicate a possible evolutionary connection between the different classes.

\section{Sample selection and preparation}

To investigate the relationship between star forming galaxies (SFG) and AGNs, we selected a sample of emission line galaxies from the Sloan Digital Sky Survey Data Release 7 (SDSS DR7, see Abazajian et al. 2009). We searched the spectra of galaxies with ionized gas components in the nucleus. The classification of these objects according to their ionization source led to the introduction of a diagnostic technique, which could successfully manage line emitting sources, including both Type 1 and Type 2 AGNs. Our choice was the diagram plotting $\log \left(F_{[\mathrm{OII}] 3727} / F_{[\mathrm{OIII}] 5007}\right)$ against $\log \left(F_{[\mathrm{OI}] 6300} / F_{[\mathrm{OIII}] 5007}\right)$ (hereafter the $O_{123}$ diagram). This instrument combines two di- 

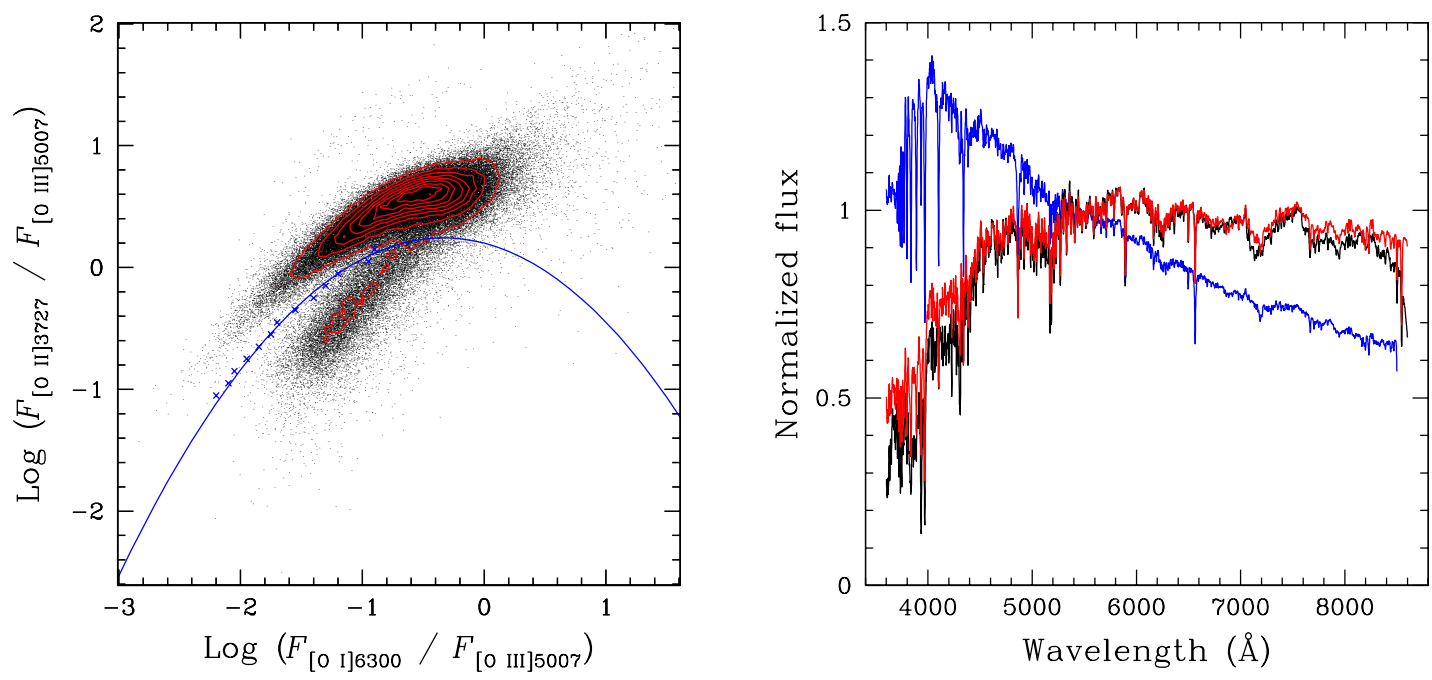

Figure 1: Left - $O_{123}$ diagram of SDSS emission line galaxies with $S / N_{[\mathrm{OI}] 6300}>3$. Red curves are isodensity contours in steps of 55 objects per unit surface, blue crosses locate the minimum densities in $0.1 \mathrm{dex}$ wide horizontal strips between the star forming and active galaxy distribution, while the blue curve is the best polynomial fit to the density minima. Right - Average stellar continua from the circum-nuclear spectra of NGs (black), S2Gs (red) and SFGs (blue), normalized at $5500 \AA$ A.

agnostic ratios that express, respectively, the ionization degree and the hardness of the ionizing spectrum at large radii from the source (Kewley et al. 2006; Shields \& Filippenko 1990). Requiring a $S / N>3$ for the [O I] $\lambda 6300$ emission line, we selected 119226 objects that we plot in the left panel of Fig. 1. We notice that objects powered by non-thermal sources lie on the lower sequence of the diagram, while the upper one is populated by star forming galaxies. By taking into account only the objects in the lower region marked by the curve, we isolated a sample of approximately 16000 objects that consists of 60 per cent Seyfert galaxies, including both broad and narrow line sources, 31 per cent composite Seyfert - H II galaxies, 4 per cent LINERs, and 5 per cent SFGs. A detailed classification of the narrow line emitting objects can be performed by application of the classic Veilleux-Osterbrock diagnostic diagrams. Before proceeding, however, we have to account for the stellar contributions, which significantly affect the intensities of recombination lines like $\mathrm{H} \alpha$ and $\mathrm{H} \beta$. These contributions were estimated with a spectral synthesis technique, based on the STARLIGHT code (Cid Fernandes et al. 2005), using a set of 92 simple stellar population spectra (SSP), obtained combining 23 ages (from $10^{6} \mathrm{yr}$ up to $13 \cdot 10^{9} \mathrm{yr}$ ) and 4 metallicities $(Z=$ $0.004,0.008,0.020$, and 0.050 ) from the library of Bruzual \& Charlot (2003). A threshold value of $S / N \geq 10$ at $5500 \AA$ granted the possibility to apply the fitting procedures with reliable results.

Since we are interested in the properties of the circum-nuclear regions of the galaxies, we restricted the sample to objects in the redshift range of $0.04 \leq z \leq 0.08$, where the SDSS fiber aperture covers a region of $2.4-4.8 \mathrm{kpc}$ in radius. In this study we considered only Seyfert 2 galaxies (S2G), collecting 2153 objects. A remaining set of 1302 SFGs was identified and an additional collection of 3000 galaxies without detected emission lines, in the same redshift range 

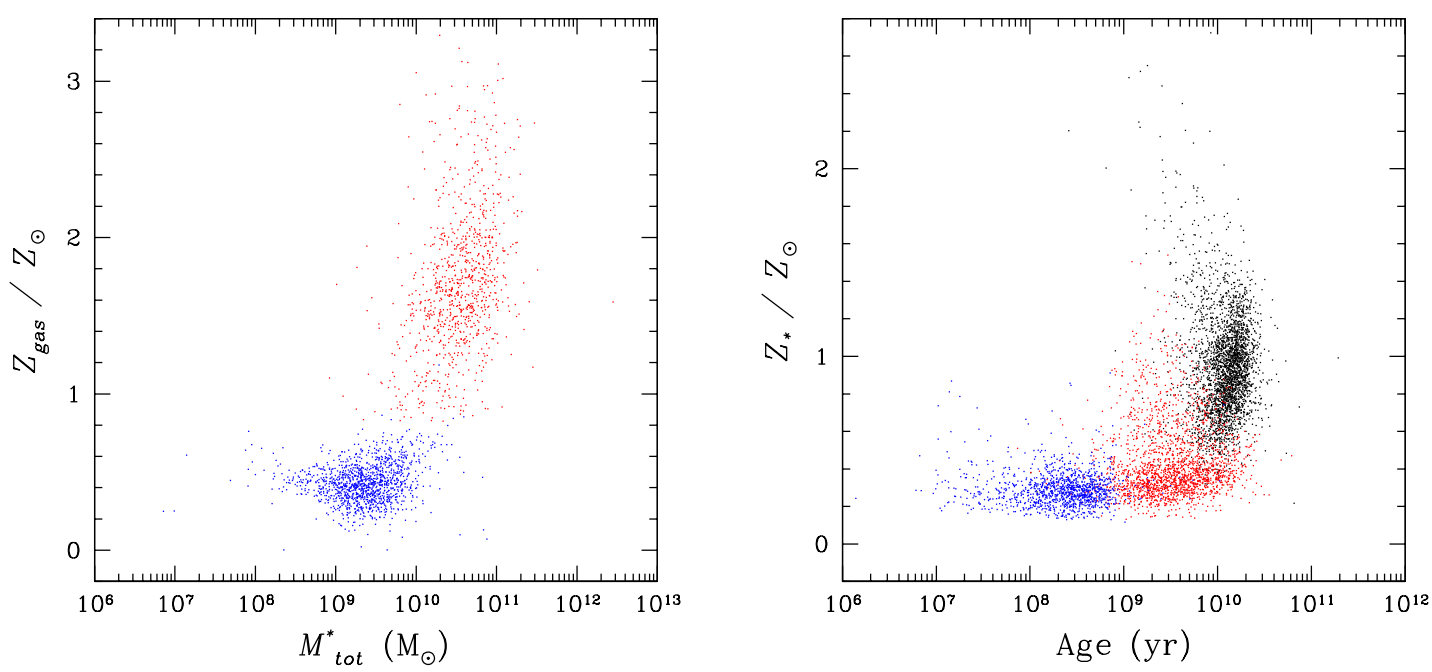

Figure 2: Left - Gas phase metallicities as a function of stellar masses for SFGs (blue points) and S2Gs (red points). Right - Stellar metallicities and ages for SFGs (blue), S2Gs (red) and NGs (black).

and above the $S / N=10$ threshold, formed our normal galaxy (NG) sample. The average spectra of the stellar populations observed in these groups of galaxies are compared, after normalization to the specific flux at $5500 \AA$, in the right panel of Fig. 1. The spectra of SFGs are obviously different, with respect to the other classes, due to the presence of young hot stars, which dominate the emission, yielding a blue peaked spectral energy distribution (SED). The spectra of NGs and S2Gs, on the other hand, are remarkably similar and characteristic of an evolved stellar population. In spite of the comparable SEDs, however, S2Gs show on average a $4000 \AA$ break that is clearly shallower than the corresponding feature of NGs. It becomes therefore evident that an excess of hot stars, specifically belonging to the spectral class A, populates the circum-nuclear regions of active galaxies.

\section{Results and discussion}

Based on the average spectral properties of stellar populations, our sample suggests that nuclear activity occurs at some intermediate stage, between a star formation process and the subsequent quiescent evolution. However, because of the well known problem of the age - metallicity degeneracy, a young, metal-rich stellar population could be confused with an old, metal-poor one. In order to solve this problem, we investigated the relationship existing between the stellar component and the inter-stellar medium (ISM) of our galaxies. Due to mass exchanges between stars and diffuse ISM, the chemical evolution connected to stellar activity propagates in the gas, where it affects the metallicity of subsequent stellar generations. In the case of SFGs, we can estimate the gas phase metallicity by means of spectral indices combining different emission lines (Pagel et al. 1979; Pilyugin \& Thuan 2005). For S2Gs the situation is further complicated by the non-thermal shape of the ionizing spectrum and proper photo-ionization models must be calculated to fit the 
observed emission line spectra (La Mura et al. 2012). All models agree appreciably well to predict a metallicity range of $1.0 Z_{\odot} \leq Z \leq 2.5 Z_{\odot}$ for the ISM of S2Gs. The high metallicity of this gas indicates that the AGN fuel is an evolved material. This, in turn, supports the idea that the gas has a local origin in the vast majority of the observed S2Gs. On the other hand, as we show in Fig. 2 (left), a substantially different situation applies to the chemical properties of the ISM in SFGs. In general, these objects are rather characterized by an ISM with a sub-solar chemical composition, suggesting that their on-going star formation is a first generation process. This result is also confirmed by the evidence that stellar masses in galaxies hosting nuclear star formation are generally lower than in S2Gs.

Despite the different chemical compositions of the gas, the average metallicity detected in the stellar populations in SFGs and S2Gs is mostly similar, but with a slight over-abundance of heavy elements in the second group (Fig. 2 right). The stellar metallicity in the SFG sample is low, with no appreciable dependence on the age of the populations, as we would expect for a generation of stars originated by an unpolluted medium. Some S2Gs, on the contrary, contain high metallicity population components, which represent a hint of further star formation in a chemically enriched medium, bringing such objects on the same range of metallicities, commonly observed in the circum-nuclear regions of NGs. This suggests that SFGs and S2Gs host stellar populations mainly formed in a pristine inter-stellar gas, but, as it is also suggested by the average spectral properties illustrated in Fig. 1 (right), the process occurred earlier in $\mathrm{S} 2 \mathrm{Gs}$, where the O/B spectral class stars have already evolved off the main sequence.

This scenario is consistent with the relationship between the stellar population ages in the circum-nuclear regions of the selected galaxies and their total stellar masses. As it is shown in Fig. 3 (left), SFGs and S2Gs lie on a well defined sequence of increasing nuclear population age for larger total mass. The sequence has a high mass break and the range of masses, where AGN activity is observed, is also populated by NGs. The total stellar mass, therefore, increases regularly with age, through the star forming and nuclear activity stages. Later on, the stellar mass assembly is stopped and galaxies evolve as quiescent objects, dominated by old populations. Before drawing any conclusions, however, we have to consider the possible biases within our sample. In particular, the choice of a S/N limiting threshold reflects in the selection of more luminous objects. SFGs are not particularly prone to this problem, because the high luminosity of their young stellar populations enables us to detect objects with fairly small masses, but for S2Gs and NGs this could be a real issue. If we estimate the mass distribution of the SDSS line emitting sources, which, in the considered redshift range, fall in the AGN region of the $O_{123}$ diagram, however, it turns out that a $S / N>10$ threshold is not systematically excluding low mass objects from the sample, thus the observed mass range of S2Gs is a real feature in the considered volume. The NG sample, on the other hand, experiences a quite different situation. In this case, we are not able to include very low mass quiescent objects, but the issue is not particularly critical for our interests, because the artificial mass cut-off falls below the transition region between star forming and active galaxies.

In addition to the differences that we appreciated on the basis of our averaged analysis, the diagram plotted in the right panel of Fig. 3 illustrates the distribution of the $D_{n}(4000)$ spectral index (defined as in Kauffmann et al. 2003) as a function of the total stellar mass. SFGs and S2Gs follow a smooth sequence of regular growth in the index, with increasing host galaxy stellar mass. Quiescent galaxies, instead, are well separated from this sequence. The situation is fully consistent 

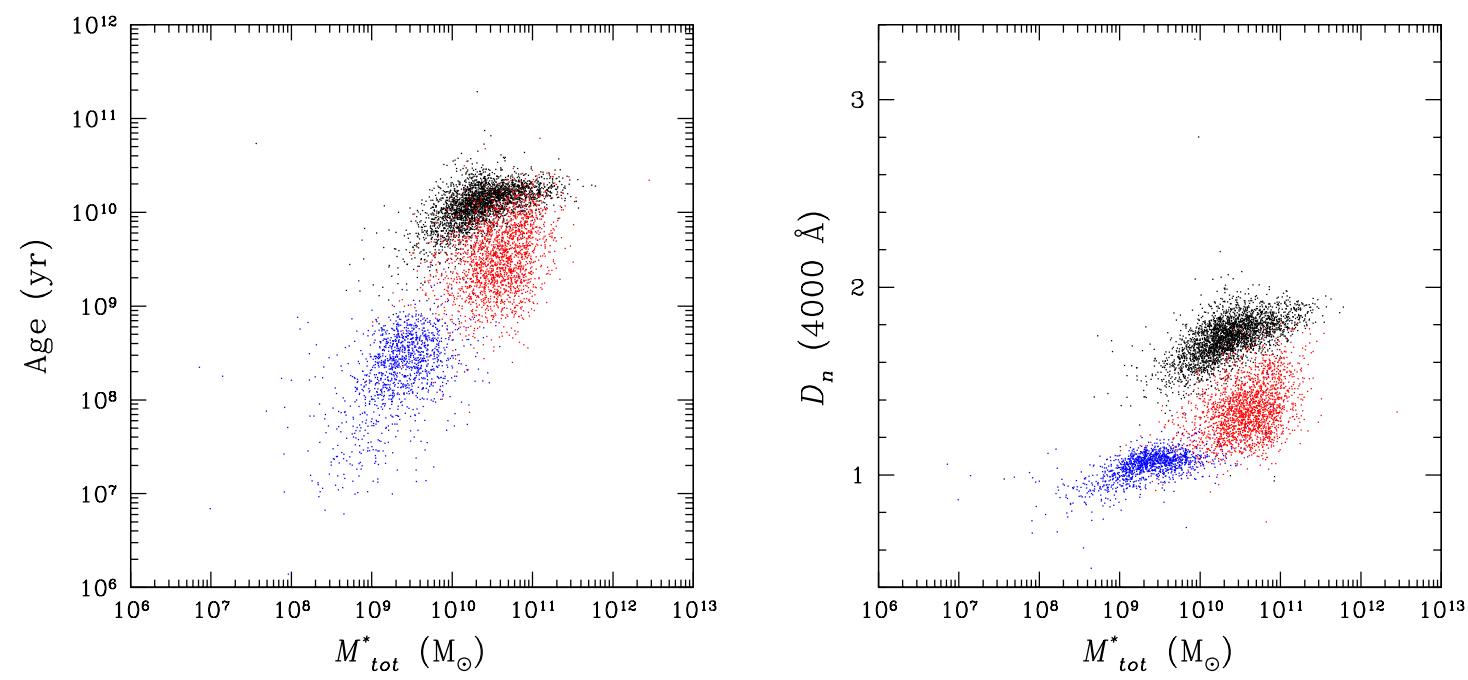

Figure 3: Left - Stellar population ages as a function of stellar masses for SFGs (blue points), S2Gs (red points) and NGs (black points). Right $-D_{n}(4000)$ as a function of stellar masses for the same groups of galaxies.

with a smooth transition of gradually increasing stellar ages in a generation of stars formed in pristine gas, as soon as we move from low mass SFGs to high mass SFGs, all the way across S2Gs. The transition region can be identified within a range of total stellar masses of $9.8 \leq \log \left(M_{\star} / M_{\odot}\right) \leq$ 10.3. The overlapping mass range may represent a region where star forming activity is most likely going to start an interplay with the AGN. Depending on the interaction between these processes, the galaxy may either stop its stellar mass assembly, eventually evolving as a low mass quiescent system, or form new generations of stars, like the high metallicity populations detected in some $\mathrm{S} 2 \mathrm{G}$ spectra of our sample, to become a high mass system. The difference between the two evolutionary possibilities probably resides in the power of the nuclear source and the way it affects the ISM in the surrounding space.

\section{References}

[1] K. N. Abazajian et al., The Seventh Data Release of the Sloan Digital Sky Survey, ApJS 182 (2009) 543 [arXiv:0812.0649v2]

[2] G. Bruzual \& S. Charlot, Stellar population synthesis at the resolution of 2003, MNRAS 344 (2003) 1000 [astro-ph/0309134v1]

[3] R. Cid Fernandes, A. Mateus, L. Sodré, G. Stasińska, J. M. Gomes, Semi-empirical analysis of Sloan Digital Sky Survey galaxies - I. Spectral synthesis method, MNRAS 358 (2005) 363 [astro-ph/0412481v1]

[4] C. Feruglio, R. Maiolino, E. Piconcelli, N. Menci, H. Aussel, A. Lamastra, F. Fiore, Quasar feedback revealed by giant molecular outflows, A\&A 518L (2010) 155 [arXiv: $1006.1655 \mathrm{v} 2$ ] 
[5] G. Kauffmann et al., The host galaxies of active galactic nuclei, MNRAS 346 (2003) 1055 [astro-ph/0304239v1]

[6] L. J. Kewley, B. Groves, G. Kauffmann, T. Heckman, The host galaxies and classification of active galactic nuclei, MNRAS 372 (2006) 961 [astro-ph/ $0605681 \mathrm{v} 3$ ]

[7] G. La Mura, D. Bindoni, S. Ciroi, V. Cracco, R. D’Abrusco, P. Rafanelli, L. Vaona, The Relation between Nuclear Activity and Stellar Mass in Galaxies, MNRAS, 426 (2012) 1893

[arXiv:1210.0422v2]

[8] P. Monaco \& F. Fontanot, Feedback from quasars in star-forming galaxies and the triggering of massive galactic winds, MNRAS 359 (2005) 283 [astro-ph/ $0502145 \mathrm{v} 1$ ]

[9] B. E. J. Pagel, M. G. Edmund, D. E. Blackwell, M. S. Chun, G. Smith, On the composition of H II regions in southern galaxies. I - NGC 300 and 1365, MNRAS, 189 (1979) 95

[10] L. S. Pilyugin \& T. X. Thuan, Oxygen Abundance Determination in H II Regions: The Strong Line Intensities-Abundance Calibration Revisited, MNRAS, 631 (2005) 231

[11] J. C. Shields \& A. V: Filippenko, Emission-line properties of the composite Seyfert/Starbust galaxy IC 5135, AJ 100 (1990) 1034

[12] Y. Taniguchi, Starburst-AGN Connections from High Redshift to the Present Day, in proceedings of the IAU 8th Asian-Pacific Regional Meeting, ASPC 289 (2003) 353 [astro-ph/0211161v1]

[13] S. Veilleux \& D. E. Osterbrock, Spectral classification of emission-line galaxies, ApJS 63 (1987) 295 